Original Research

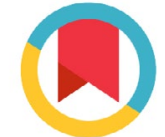

Check for updates
\# Contributed equally

* For correspondence: ngocvu@sci.edu.vn vbngoc@hcmus.edu.vn

Competing interests: The authors declare that no competing interests exist.

Received: 10 December 2017 Accepted: 29 January 2018 Published: 26 February 2018

Copyright The Author(s) 2018. This article is published with open access by BioMedPress

This article is distributed under the terms of the Creative Commons Attribution License (CC-BY 4.0) which permits any use, distribution, and reproduction in any medium, provided the original author(s) and the source are credited.

\section{Engineered cartilage tissue from biodegradable Poly( $\varepsilon$-caprolactone) scaffold and human umbilical cord derived mesenchymal stem cells}

\author{
Phuc Dang-Ngoc Nguyen1,\#, Ngoc Bich Vu1,2,\#,* iD, Ha Thi-Ngan Le 1,2, \\ Thuy Thi-Thanh Dao1,2, Long Xuan Gia4, Phuc Van Pham¹,2,3 iD
}

\author{
${ }^{1}$ Stem Cell Institute, University of Science, VNUHCM, Ho Chi Minh City, Vietnam \\ 2Laboratory of Stem Cell Research and Application, University of Science, VNUHCM, Ho \\ Chi Minh City, Vietnam \\ ${ }^{3}$ Faculty of Biology and Biotechnology, University of Science, VNUHCM, Ho Chi Minh \\ city, Vietnam \\ 4Institute of Applied Mechanics and Informatics - Vietnam Academy of Science and \\ Technology, Viet Nam
}

\begin{abstract}
Introduction: Cartilage injury is the most common injury among orthopedic diseases. The predominant treatment for this condition is cartilage transplantation. Therefore, production of cartilage for treatment is an important strategy in regenerative medicine of cartilage to provide surgeons with an additional option for treatment of cartilage defects. This study aimed to produce in vitro engineered cartilage tissue by culturing and differentiating umbilical cord derived mesenchymal stem cells on biodegradable Poly $(\varepsilon-$ caprolactone) (PCL) scaffold. Methods: Human umbilical cord derived mesenchymal stem cells (UCMSCs) were isolated and expanded according to previous published protocols. UCMSCs were labeled with CD90 APC-conjugated monoclonal antibody (CD90-APC) and then seeded onto porous PCL scaffolds. Cell adhesion and proliferation on PCL scaffolds were evaluated based on the strength/signal of APC, MTT assays, and scanning electron microscopy (SEM). The chondrogenic differentiation of UCMSCs on scaffolds was detected by Alcian Blue and Safranin $O$ staining. Results: The results showed that UCMSCs successfully adhered, proliferated and differentiated into chondroblasts and chondrocytes on PCL scaffolds. The chondrocyte scaffolds were positive for some markers of cartilage, as indicated by Alcian Blue and Safranin $\bigcirc$ staining.
\end{abstract}


Conclusion: In conclusion, this study showed successful production of cartilage tissues from UCMSCs on PCL scaffolds.

\section{Keywords}

3D scaffold, Chondrocytes, Human umbilical cord, Mesenchymal stem cells, Poly( $(\varepsilon-$ caprolactone) (PCL)

\section{Introduction}

Cartilage lesions are a common problem in the orthopedic practice. In recent years, cartilage lesions (or defects/injury) have been treated by various different strategies, including microfracture (Sledge, 2001; Steadman et al., 1999), autologous chondrocyte implantation (ACI) (Brittberg et al., 1994; Podskubka et al., 2006), osteochondral allograft transplantion surgery (OATS) (Hangody et al., 1997; Okamoto et al., 2007), and stem cell transplantation (Bui et al., 2014; Nguyen et al., 2017). These treatments have demonstrated some promising results; however, they have also shown some limitations, especially the lack of cartilage tissue for transplantation. Therefore, this study aimed to investigate a potential approach to produce engineered cartilage tissue, using stem cells seeded on scaffolds.

Mesenchymal stem cells (MSCs) have been used to produce cartilage in vitro in previous studies (Marmotti et al., 2017). However, there are various kinds of MSCs which can be utilized for differentiation into chondrocytes in vitro. Notably, umbilical cord derived MSCs (UCMSCs) display some useful properties compared to bone marrow derived MSCs or adipose tissue derived MSCs, such as the abundant source of umbilical cord and the strong proliferation and easy isolation of UCMSCS (Ding et al., 2015). Moreover, it has previously been reported that UCMSCs can adhere to scaffolds based on architecture and the interactions between cell membranes and scaffold surfaces (Guarino et al., 2011).

Poly( $\varepsilon$-caprolactone) (PCL) is a biodegradable polyester (Labet and Thielemans, 2009). PCL scaffolds are a bio-scaffold with robust biocompatibility and biodegradation, especially with respect to degradation as it takes about 3 years longer than other scaffolds (Woodruff and Hutmacher, 2010). With these characteristics, PCL is thought to be an essential material for cartilage regeneration in tissue engineering applications.

Therefore, this study aimed to produce engineered cartilage in vitro from UCMSCs using PCL scaffolds (and chondrogenesis medium). 


\section{Materials-Methods}

\section{Poly( $\varepsilon$-caprolactone) scaffold}

PCL scaffolds were provided by the Institute of Applied Mechanics and Informatics (Academy of Science and Technology, Vietnam). They have structural pores ranging from 200-300 $\mu \mathrm{m}$ in diameter. PCL scaffolds were sterilized by soaking in alcohol (96\%) for one day. Next, the scaffolds were washed in phosphate buffered saline (PBS) and air-dried.

\section{Human umbilical cord derived mesenchymal stem cells}

The frozen UCMSCs were produced by our Institute as previously published (Van Pham et al., 2016a; Van Pham et al., 2016b). The frozen UCMSCs were thawed at $37 \circ \mathrm{C}$ for 1-2 minutes and then centrifuged at $1000 \mathrm{rpm}$ for 5 minutes to eliminate the cryopreservation medium. The supernatants were then removed and the pellet was re-suspended with $5 \mathrm{ml}$ MSCCult medium containing DMEM/ $\mathrm{F}-12,10 \% \mathrm{FBS}, 10 \mathrm{ng} / \mathrm{ml} \mathrm{EGF}, 10 \mathrm{ng} / \mathrm{ml} \mathrm{FGF}, 1 \%$ antibiotic-antimycotic (all reagents were purchased Thermo Fisher Scientific, Waltham, MA). UCMSCs were cultured in T-25 flasks and incubated at $37{ }^{\circ} \mathrm{C}, 5 \% \mathrm{CO}_{2}$. The medium was changed every 3 days.

MSC phenotype was characterized by flow cytometry using the FACSCalibur machine (BD Biosciences, Franklin Lakes, NJ). UCMSCs were stained with antibodies, including CD14-FITC, CD73-FITC, CD90-FITC, CD105-PE, and HLADR-FITC antibodies (purchased from Santa Cruz Biotechnology, Dallas, TX), and CD34-FITC and CD45-FITC antibodies (BD Biosciences, Franklin Lakes, NJ). Cells were stained with appropriate antibodies for $20 \mathrm{~min}$ at room temperature. Then, the stained cells were analyzed by BD CellQuest Pro software with a minimum of 10,000 events.

The in vitro ability of UCMSCs to differentiate into adipocytes, osteoblasts and chondroblasts were evaluated by induced media. For adipocyte differentiation, UCMSCs were incubated in DMEM/F12 medium supplemented with 10\% FBS, $1 \%$ of 100X antibiotic-antimycotic, $10 \mathrm{mM}$ mexamethasone, $2.79 \mathrm{mM}$ indomethacin, $5 \mathrm{mg} / \mathrm{ml}$ insulin, and $0.5 \mathrm{M}$ of 1-Methyl-3-isobutylxanthine (IBMX) (Sigma Aldrich, Louis St, MO). To induce differentiation into osteoblasts and chondroblasts, UCMSCs were treated according to the StemPro $®$ Osteogenesis and Chondrogenesis Differentiation Kits (Thermo Fisher Scientific, Waltham, MA), respectively. After 20-30 days of induction, the differentiated cells were assessed by staining with Oil Red $O$ for adipocytes, Alizarin Red $S$ for osteoblasts, and Alcian Blue for chondroblasts (all reagents were purchased from Sigma Aldrich).

\section{Cell adhesion on PCL scaffolds}


CD90 APC-labeled UCMSCs (UCMSCs-CD90) were seeded onto PCL scaffolds at a density of about $10^{5}-10^{6}$ cells/ml. UCMSCs-CD90 adhesion on PCL scaffolds was recorded by measurement of fluorescent signal under a fluorescent microscope at excitation wavelength 594 and $633 \mathrm{~nm}$.

\section{Cell proliferation on PCL scaffolds}

MTT assay was performed to evaluate the proliferation of UCMSCs on PCL scaffolds. UCMSCs were seeded at a density of $1.2 \times 10^{4}$ cells/PCL scaffold (size $3 \times 4 \mathrm{~mm}$ ). After 3, 7 and 14 days, fresh medium was replaced, then $10 \mu \mathrm{l}$ of MTT solution $(0.45 \mathrm{mg} / \mathrm{ml})$ was added. These samples were incubated at $37 \circ \mathrm{C}, 5 \%$ $\mathrm{CO}_{2}$. After 4 hours, MTT solution was removed and $100 \mu \mathrm{L}$ DMSO was added (Merck, Germany) to dissolve the formazan crystal. The optical density (OD) values were recorded at $595 \mathrm{~nm}$ by a DTX-880 system (Beckman Coulter, Brea, CA).

\section{Cell -seeded PCL surface structure change}

Cell- seeded PCL surface structure was analyzed by using a scanning electron microscope (SEM) (Hitachi, Tokyo, Japan). UCMSCs (cultured on PCL) was fixed in $4 \%$ paraformaldehyde (PFA) (Merck, Kenilworth, NJ) for 30 minutes. This sample was compared to $\mathrm{PCL}$ without cells to determine the changing structure of scaffolds in the presence or absence of seeded cells.

\section{Cartilage differentiation of UCMSCs on scaffolds and analysis of aggrecan and glycosaminoglycan expression}

The UCMSCs on PCL scaffolds were induced to differentiate into chondrocytes by a commercial induction medium (Chondrogenesis Differentiation Kit), according to the company guidelines. These scaffolds were analyzed after 21 days by Alcian Blue and Safranin O staining (Merck, Kenilworth, NJ). The scaffolds were taken out of medium and fixed in 4\% PFA for 30 minutes then rinsed with PBS and soaked in staining solution. For Alcian Blue staining, the scaffolds were stained for 30 minutes then washed with de-staining solution $\left(3 \mathrm{C}_{2} \mathrm{H}_{5} \mathrm{OH}: 2 \mathrm{CH}_{3} \mathrm{COOH}\right)$, and washed with $\mathrm{PBS}$ again to remove all acidic solution. The scaffolds were finally observed under a microscope to determine whether or not aggrecan proteins were expressed. For Safranin $O$ staining, the scaffolds were stained for 10 minutes then washed with $90-95 \%$ alcohol. The scaffolds were washed with PBS again and observed under a microscope to determine the presence/expression of glycosaminoglycans (GAGs).

\section{Statistical Analysis}

From the data, mean \pm SD were calculated. GraphPad Prism Software (San Diego, CA, USA) was used to calculate statistical significance of the results. The difference is considered as significant difference if $p<0.05$. 


\section{Results}

\section{Characterization of UCMSCs}

After thawing for 3 days, UCMSCs were adherent and had spread on the culture surface. They exhibited fibroblast-like morphology (Fig. 1A). The abilities of UCMSCs to differentiate in vitro into mesenchymal cells were evaluated by inducing UCMSCs into osteocytes, adipocytes and chondrocytes in 20-30 days. The results showed that in osteogenesis medium, UCMSCs could accumulate calcium, which can bind to Alizarin Red and form red complexes (Fig. 1B). In adipogenesis medium, UCMSCs formed intracellular lipid droplets that were positive with Oil Red staining assay (Fig. 1C). In chondrogenesis medium, UCMSCs expressed aggrecans which can link with Alcian Blue to form blue complexes (Fig. 1D). The cells maintained the surface markers of MSCs- they were positive for CD73, CD90, and CD105, and negative for CD14, CD34, CD45, and HLA-DR (Fig. 1E-M).
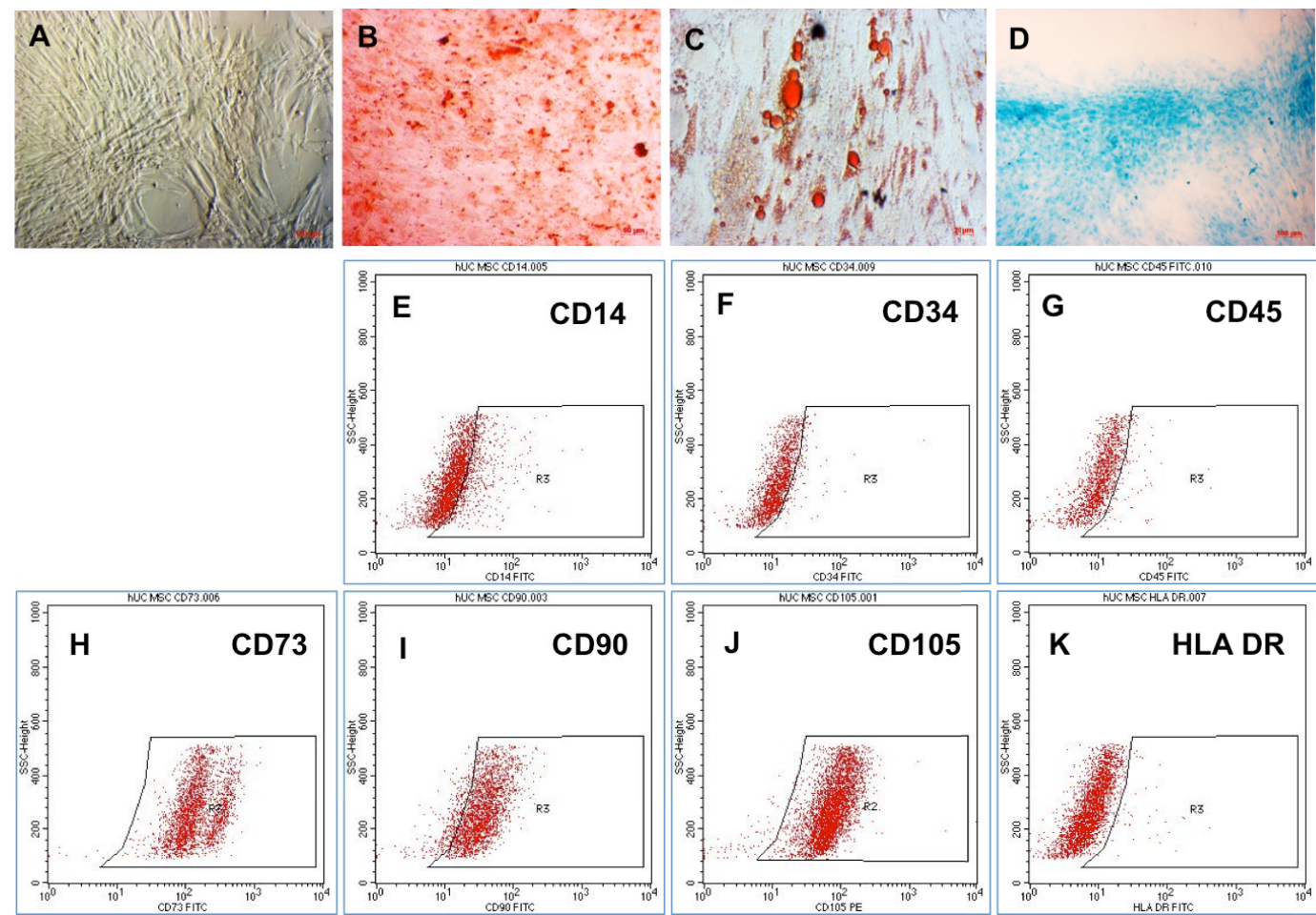

Figure 1. Phenotype of UCMSCs after thawing and proliferation. UCMSCs exhibited the criteria of MSCs: fibroblast-like shape $(\mathbf{A})$; in vitro differentiation into osteoblasts (B), adipocytes (C), and chondrocytes (D); and expression of CD73 (H), CD90 (I) and CD105 (J) markers, but absence of CD14 (E), CD34 (F), CD45 (G) and HLA-DR (K) markers. 


\section{Adhesion of UCMSCs on PCL scaffolds}

After 3 days of culture, UCMSCs adhered and expanded on the scaffold surface (Fig. 2B). UCMSCs adhered and formed cell clusters at the pores of the PCL scaffolds after 7 days (Fig. 2C). From 21 to 70 days, UCMSCs developed and grew to $80-90 \%$ confluence in the pores of the scaffolds (Fig. 2D). These observations showed that UCMSCs adhered and proliferated well on the PCL scaffold surface. The existence of UCMSCs on the PCL surface was confirmed by SEM capture. As represented in Figure 3, UCMSCs fully covered the surface of PCL (Fig. 3).
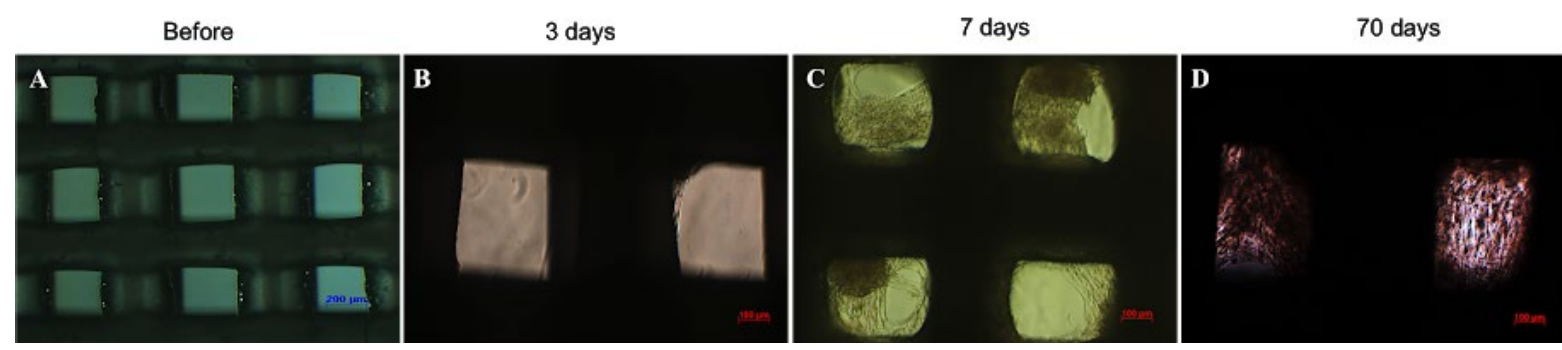

Figure 2. Adhesion of UCMSCs on PCL scaffold surface. The UCMSCs started to attach to the scaffold surface after 3 days of culture $(\mathbf{B})$; the number of UCMSCs rapidly proliferated on the surface to fill up the pores of scaffold after 7 days $(\mathbf{C})$; and cell density was markedly increased after 70 days (D).

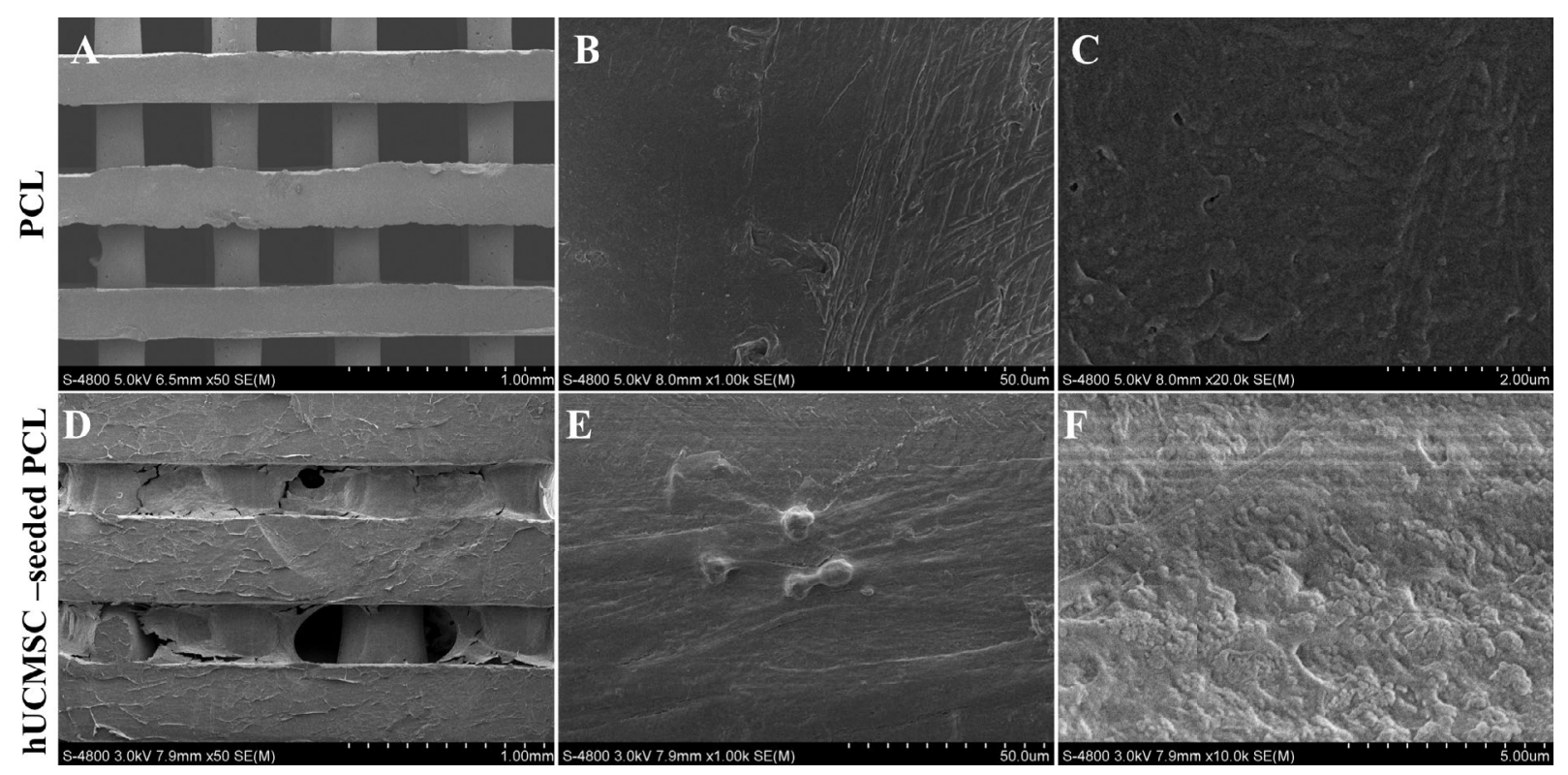

Figure 3. SEM captures of UCMSC adherence on surface of PCL scaffolds.

The structure and surface of PCL scaffolds were observed under SEM at different magnifications: $1 \mathrm{~mm}(\mathbf{A}), 50 \mathrm{um}(\mathbf{B})$, and 2 um (C). After 7 days of culture, UCMSCs adhered onto the surface of PCL (D), and exhibited mitosis (E) and high confluence $(\mathbf{F})$. 


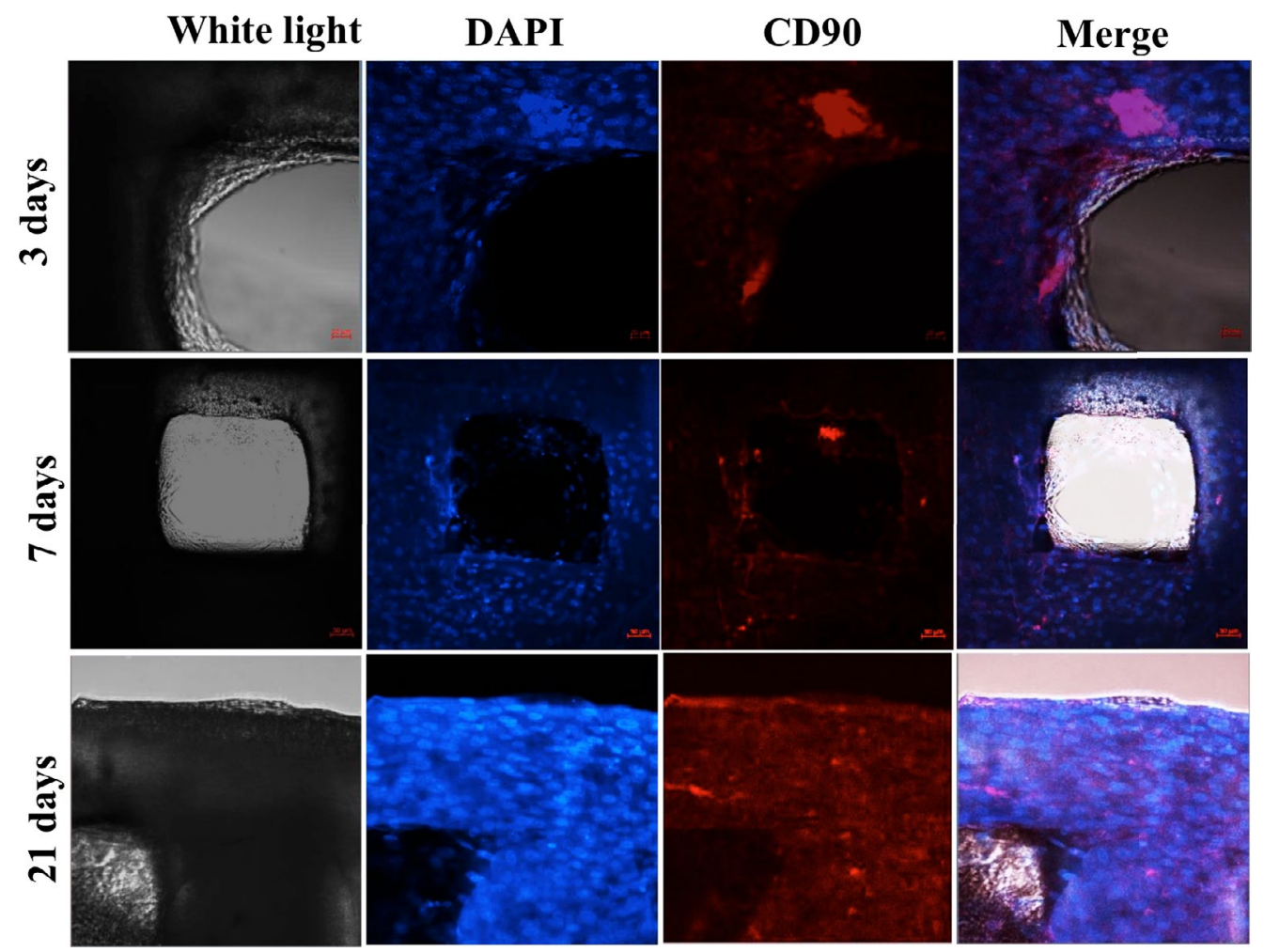

Figure 4. Proliferation of UCMSCs was evaluated by cellular nuclei staining with DAPI and CD90 (MSC marker). There was a significant increase of DAPI signal and CD90-APC expression from day 3 to day 7; day 21 showed significant proliferation of UCMSCs on the PCL surface (Magnification $\times 20$ ).

\section{UCMSC proliferation on PCL scaffolds}

After 3 days of culture, UCMSCs began to attach on the surface of PCL scaffolds in the pores. After 7 days, spreading UCMSCs occurred, covering numerous scaffold pores and forming cell clusters. The increase of UCMSCs showed that UCMSCs proliferated from day 3 to day 21 (Fig. 4). MTT assay was used as a quantitative method to monitor cell proliferation on PCL scaffolds. From day 3 to day 7 , there was a significant increase of OD values. From day 7 to 14 , UCMSCs continuously proliferated at a slower rate. Indeed, the OD values at day 14 showed a non-significant increase (Fig. 5).

\section{UCMSC differentiation into chondrocytes on PCL scaffolds}

After 21 days of differentiation into chondrocytes, UCMSCs changed their shape and showed similar morphology to chondrocytes (rounded and smaller). The cells were strongly positive for Alcian Blue and Safranin O staining (Fig. 6), when fixed and stained with these dyes. 


\section{Biomedical}

Research \& Therapy
ISSN: $2198-4093$

www.bmrat.org

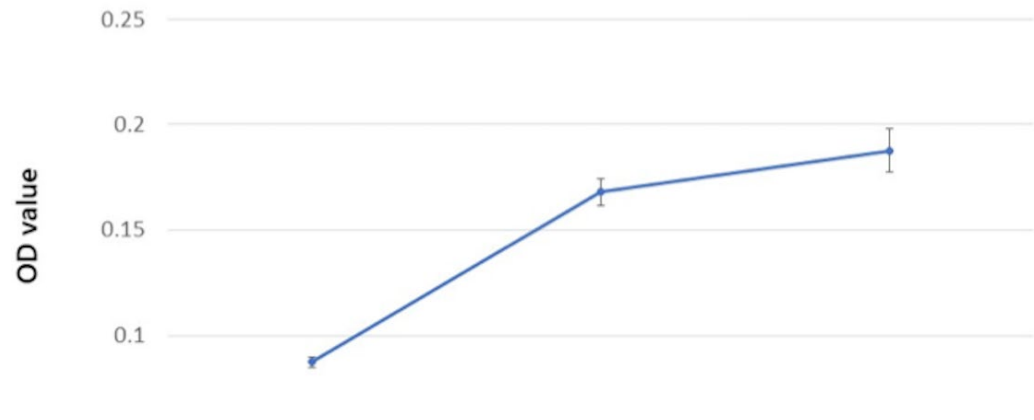

0.05

Day 3

Day 7

Day 14

Day

Figure 5. UCMSC proliferation assessed by MTT assay. From day 3 to day 7 , the OD values significantly increased, demonstrating that UCMSCs rapidly proliferated from day 3 to day 7 ; however, from day 7 to day 14 this increase was non-significant.

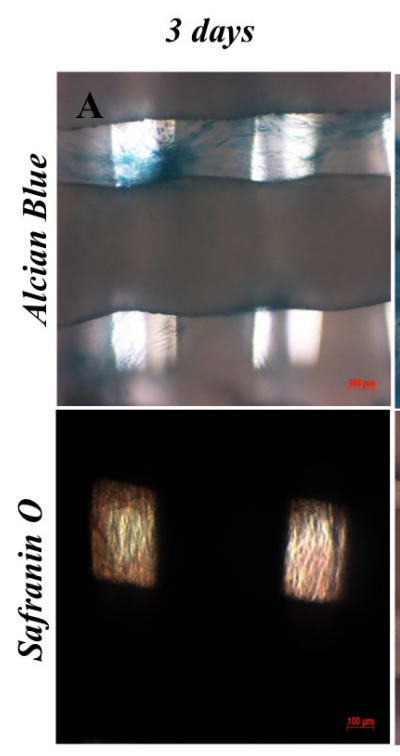

7 days

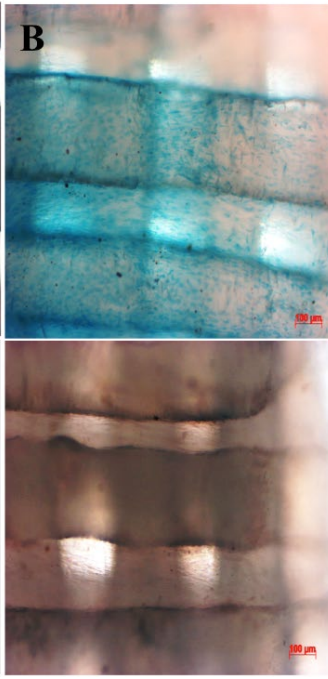

14 days

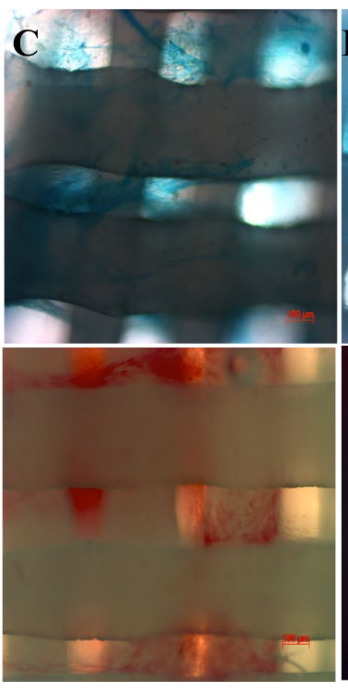

21 days

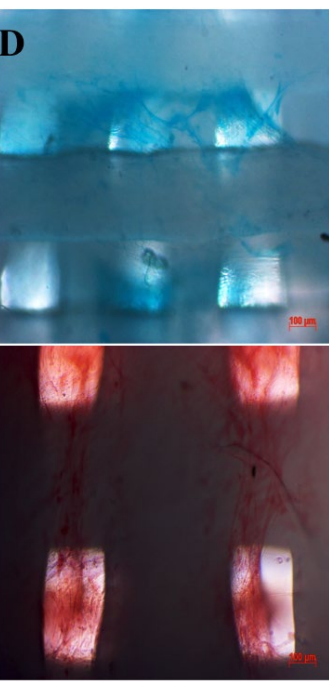

Figure 6. Differentiation of UCMSCs on PCL scaffolds. After 3 days of induction, the staining results showed that UCMSCs were weakly positive for Alcian blue and Safranin O staining. However, after 7, 14 and 21 days of induction, UCMSCs showed strongly positive staining with these dyes. 


\section{Discussion}

Engineered cartilage or cartilage engineering is a promising approach to produce adequate cartilage tissues for orthopedic applications, especially for cartilage injury repair. This study aimed to produce engineered cartilage in vitro using UCMSCs and PCL scaffolds. In this study, we showed preliminary success of producing cartilage tissues when culturing UCMSCs on PCL scaffolds, then differentiating them into chondrocytes using suitable inducing medium.

In the first assay, UCMSCs proliferated well and were confirmed to exhibit MSC phenotypes. Indeed, they exhibited the minimal criteria for MSCs, according to the suggested guidelines of the International Society of Cellular Therapy (Dominici et al., 2006). More importantly, these cells were confirmed for their chondrocyte differentiation potential in monolayer culture conditions in vitro. They showed successful differentiation into chondrocytes.

Based on this ability, in the next experiment UCMSCs were cultured on PCL scaffolds (the porous structures were made from PCL material). UCMSCs showed they could adhere and proliferate on the surface of these scaffolds. Indeed, this was evident from visualization of UCMSCs on scaffold surface by inverted light microscopy as well as SEM. Data from both microscopes demonstrated that UCMSCs not only strongly adhered but also underwent mitosis and showed robust proliferation on the scaffold structures.

Cellular nuclei staining with DAPI in combination with CD90 marker demonstrated the proliferation of UCMSCs on PCL scaffolds yet also the maintenance of their stemness during the culture. The adherence and proliferation of UCMSCs was also confirmed by the MTT assay. Similarly, Nirmal et al. (2013) and Gauthaman et al. (2011) successfully cultured MSCs in PCL materials and scaffolds (Gauthaman et al., 2011; Nirmal and Nair, 2013). In a recent study, Xue et al. (2017) also showed that various kinds of MSCs, including UCMSCs, bone marrow MSCs and adipose tissue derived MSCs, can adhere and proliferate on PCL (Xue et al., 2017).

After UCMSCs proliferated to about $70-80 \%$ confluence on the PCL scaffold surface, they were induced to differentiate into chondrocytes in the same medium conditions that were used to differentiate them in monolayer culture. The results confirmed that after 3 days of induction, UCMSCs on PCL scaffolds started to produce and accumulate GAG proteins that could be detected by Alcian Blue and Safranin $O$ staining. Indeed, Safranin $O$ is a dye which can bind strongly to sulfate groups $\left(\mathrm{SO}_{4}{ }^{2-}\right)$ of $\mathrm{GAGs}$, which has a specific role in chondrogenesis. GAG sulfates appeared in the early phase of chondrocyte formation (Demoor et al., 2014). Alcian Blue is a dye that can bind to aggrecans, which appears at the early phase of chondrogenesis. The weakly positive results demonstrated that the induced cells began secreting aggrecans, which is a sign of chondrocyte formation. 
From day 7 to day 21, chondrogenesis was more robust- as indicated by stronger positive Alcian Blue and Safranin $O$ staining. Using different scaffolds (alginate gels), Xu et al. (2008) also showed similar results that MSCs could be differentiated into chondrocytes after 6 days of induction (Xu et al., 2008). In 2013, Nirmal et al. showed that umbilical cord matrix derived MSCs could proliferate and become differentiated into chondrocytes on polyvinylalcohol-PCL (PVA-PCL) scaffolds (Nirmal and Nair, 2013). They also found that the growth factor combination of tumor growth factor beta 3 (TGF-B3) and BMP-2 was more effective for chondrogenesis (Nirmal and Nair, 2013). Recently, Xue et al. (2017) showed that UCMSCs, bone marrow derived MSCs and adipose tissue derived MSCs could all be differentiated into osteoblasts on PCL scaffolds (Xue et al., 2017).

\section{Conclusion}

Cartilage tissue is used frequently in orthopedic surgery, especially for injured cartilage replacement. The limitation of cartilage tissue resources and donors makes it a challenge to repair cartilage injury. Although stem cell therapy can provide some benefits for patients with cartilage injury, due to the impact of cartilage degeneration stem cell therapy can barely regenerate injured cartilage tissue. Thus, cartilage engineering is the new and potentially effective approach to develop cartilage tissue for orthopedic surgery. In this study, we showed some success with producing cartilage tissue by culture and differentiation of umbilical cord derived mesenchymal stem cells on PCL scaffolds using suitable chondrogenesis medium. The engineered cartilage tissues expressed some markers of chondrocytes, as indicated by Alcian Blue and Safranin $O$ staining. Overall, these preliminary results demonstrate that cartilage can be engineered successfully in vitro using UCMSCs on PCL scaffolds. This approach may be a promising method to produce cartilage tissue for orthopedic diseases.

\section{Abbreviations}

PACl Autologous Chondrocyte Implantation

CD Cluster of Differentiation

EGF Epidermal Growth Factor

FBS Fetal Bovine Serum

GFG Fibroblast Growth Factor GAGs Glycosaminoglycans

HLA-DR Human Leukocyte Antigen - antigen D Related

IBMX 1-Methyl-3-isobutylxanthine

OATS Osteochondral Allograft Transplantion Surgery 
OD Optical Density

PCL Poly( $\varepsilon$-caprolactone)

SD Standard Deviation

SEM Scanning Electron Microscope

UCMSCs Umbilical Cord Derived Mesenchymal Stem Cells

OATS Osteochondral Allograft Transplantion Surgery

\section{Acknowledgements}

This research is funded by Vietnam National University Ho Chi Minh City (VNUHCM) under grant number C2017-18-24 and TX2017-18-02.

\section{Author Contribution}

NBV and PVP were responsible for suggesting the idea for this study, creating the experiment design. NVB and PDNN were responsible for analyzing the characterization of MSCs, the adhesion of MSC on $\mathrm{PCL}$, the structure change of $P C L$ and the data, differentiation of MSC into cartilage, writing the result, discussing, preparing the figures, and revising the manuscript. HLTN was responsible for performing the UCMSCs cultures. TTTD was responsible for estimating the cell proliferation. LVG was prepared PCL scaffold. All authors read and approved the manuscript.

\section{References}

Brittberg, M., Lindahl, A., Nilsson, A., Ohlsson, C., Isaksson, O., \& Peterson, L. (1994). Treatment of deep cartilage defects in the knee with autologous chondrocyte transplantation. The New England Journal of Medicine, 331(14), 889-895. https:// doi.org/10.1056/NEJM199410063311401 PMID:8078550

Bui, K.H.-T., Duong, T.D., Nguyen, N.T., Nguyen, T.D., Le, V.T., Mai, V.T., Phan, N.L.-C., Le, D.M., Phan, N.K., and Van Pham, P. (2014). Symptomatic knee osteoarthritis treatment using autologous adipose derived stem cells and platelet-rich plasma: a clinical study. Biomedical Research and Therapy 1, 02-08. https://doi.org/10.15419/bmrat.v1i01.11

Demoor, M., Ollitrault, D., Gomez-Leduc, T., Bouyoucef, M., Hervieu, M., Fabre, H., . . . Galera, P. (2014). Cartilage tissue engineering: Molecular control of chondrocyte differentiation for proper cartilage matrix reconstruction. Biochimica et Biophysica Acta, 1840(8), 2414-2440. https://doi.org/10.1016/j.bbagen.2014.02.030 PMID:24608030

Ding, D.-C., Chang, Y.-H., Shyu, W.-C., \& Lin, S.-Z. (2015). Human umbilical cord mesenchymal stem cells: A new era for stem cell therapy. Cell Transplantation, 24(3), 339-347. https://doi.org/10.3727/096368915X686841 PMID:25622293

Dominici, M., Le Blanc, K., Mueller, I., Slaper-Cortenbach, I., Marini, F., Krause, D., . . . Horwitz, E. (2006). Minimal criteria for defining multipotent mesenchymal stromal cells. 
The International Society for Cellular Therapy position statement. Cytotherapy, 8(4), 315-317. https://doi.org/10.1080/14653240600855905 PMID:16923606

Gauthaman, K., Venugopal, J. R., Yee, F. C., Biswas, A., Ramakrishna, S., \& Bongso, A. (2011). Osteogenic differentiation of human Wharton's jelly stem cells on nanofibrous substrates in vitro. Tissue Engineering. Part A, 17(1-2), 71-81. https://doi.org/10.1089/ ten.tea.2010.0224 PMID:20673136

Guarino, V., Alvarez-Perez, M., Cirillo, V., \& Ambrosio, L. (2011). hMSC interaction with $\mathrm{PCL}$ and $\mathrm{PCL} /$ gelatin platforms: A comparative study on films and electrospun membranes. Journal of Bioactive and Compatible Polymers, 26(2), 144-160. https:// doi.org/10.1177/0883911511399410

Hangody, L., Kish, G., Karpati, Z., Szerb, I., and Udvarhelyi, I. (1997). Arthroscopic autogenous osteochondral mosaicplasty for the treatment of femoral condylar articular defects. A preliminary report. Knee surgery, sports traumatology, arthroscopy : official journal of the ESSKA 5, 262-267.

Labet, M., \& Thielemans, W. (2009). Synthesis of polycaprolactone: A review. Chemical Society Reviews, 38(12), 3484-3504. https://doi.org/10.1039/b820162p PMID: $\underline{20449064}$

Marmotti, A., Mattia, S., Castoldi, F., Barbero, A., Mangiavini, L., Bonasia, D. E., .. Peretti, G. M. (2017). Allogeneic Umbilical Cord-Derived Mesenchymal Stem Cells as a Potential Source for Cartilage and Bone Regeneration: An In Vitro Study. Stem Cells International, 2017, 1732094. https://doi.org/10.1155/2017/1732094 PMID:29358953

Nguyen, P. D., Tran, T. D., Nguyen, H. T., Vu, H. T., Le, P. T., Phan, N. L., . . Van Pham, P. (2017). Comparative Clinical Observation of Arthroscopic Microfracture in the Presence and Absence of a Stromal Vascular Fraction Injection for Osteoarthritis. Stem Cells Translational Medicine, 6(1), 187-195. https://doi.org/10.5966/sctm.2016-0023 PMID: $\underline{28170179}$

Nirmal, R. S., \& Nair, P. D. (2013). Significance of soluble growth factors in the chondrogenic response of human umbilical cord matrix stem cells in a porous three dimensional scaffold. European Cells \& Materials, 26, 234-251. https://doi.org/ 10.22203/eCM.v026a17 PMID:24213879

Okamoto, Y., Nakagawa, Y., Maekawa, M., Kobayashi, M., and Nakamura, T. (2007). Osteochondral grafting for treatment of a massive chondral defect in the knee of a young adult with anterior cruciate ligament deficit. Arthroscopy: the journal of arthroscopic \& related surgery : official publication of the Arthroscopy Association of North America and the International Arthroscopy Association 23, 1024.e1021-1024.

Podskubka, A., Povýsil, C., Kubes, R., Sprindrich, J., \& Sedlácek, R. (2006). [Treatment of deep cartilage defects of the knee with autologous chondrocyte transplantation on a hyaluronic Acid ester scaffolds (Hyalograft C)]. Acta Chirurgiae Orthopaedicae et Traumatologiae Cechoslovaca, 73(4), 251-263. PMID:17026884

Sledge, S. L. (2001). Microfracture techniques in the treatment of osteochondral injuries. Clinics in Sports Medicine, 20(2), 365-377. https://doi.org/10.1016/ S0278-5919(05)70311-2 PMID:11398363

Steadman, J. R., Rodkey, W. G., Briggs, K. K., \& Rodrigo, J. J. (1999). [The microfracture technic in the management of complete cartilage defects in the knee joint]. Der Orthopade, 28(1), 26-32. PMID:10081041

Van Pham, P., Truong, N. C., Le, P. T.-B., Tran, T. D.-X., Vu, N. B., Bui, K. H.-T., \& Phan, N. K. (2016a). Isolation and proliferation of umbilical cord tissue derived mesenchymal stem 
cells for clinical applications. Cell and Tissue Banking, 17(2), 289-302. https://doi.org/ 10.1007/s10561-015-9541-6 PMID:26679929

Van Pham, P., Vu, N. B., \& Phan, N. K. (2016b). Umbilical cord-derived stem cells (MODULATISTTM) show strong immunomodulation capacity compared to adipose tissue-derived or bone marrow-derived mesenchymal stem cells. Biomedical Research and Therapy, 3(6), 687-696. https://doi.org/10.7603/s40730-016-0029-1

Woodruff, M. A., \& Hutmacher, D. W. (2010). The return of a forgotten polymerPolycaprolactone in the 21st century. Progress in Polymer Science, 35(10), 1217-1256. https://doi.org/10.1016/j.progpolymsci.2010.04.002

Xu, J., Wang, W., Ludeman, M., Cheng, K., Hayami, T., Lotz, J. C., \& Kapila, S. (2008). Chondrogenic differentiation of human mesenchymal stem cells in three-dimensional alginate gels. Tissue Engineering. Part A, 14(5), 667-680. https://doi.org/10.1089/tea. 2007.0272 PMID:18377198

Xue, R., Qian, Y., Li, L., Yao, G., Yang, L., \& Sun, Y. (2017). Polycaprolactone nanofiber scaffold enhances the osteogenic differentiation potency of various human tissuederived mesenchymal stem cells. Stem Cell Research \& Therapy, 8(1), 148. https:// doi.org/10.1186/s13287-017-0588-0 PMID:28646917 